\title{
The Relation between Inflammation and Neuropsychological Test Performance
}

\author{
Valerie H. Balldin, ${ }^{1}$ James R. Hall, ${ }^{2}$ Robert C. Barber, ${ }^{3}$ Linda Hynan, ${ }^{4}$ \\ Ramon Diaz-Arrastia, ${ }^{5}$ and Sid E. O'Bryant ${ }^{6,7}$
}

${ }^{1}$ Psychology Service, South Texas Veterans Healthcare System, San Antonio, TX 78229, USA

${ }^{2}$ Department of Psychiatry \& Behavioral Health, University of North Texas Health Science Center, Fort Worth, TX 76107, USA

${ }^{3}$ Department of Pharmacology \& Neurosicence, University of North Texas Health Science Center, Fort Worth, TX 76107, USA

${ }^{4}$ Department of Clinical Sciences, University of Texas Southwestern Medical Center, Dallas, TX 75390, USA

${ }^{5}$ Department of Neurology and Neurotherapeutics, University of Texas Southwestern Medical Center, Dallas, TX 75390, USA

${ }^{6}$ Department of Internal Medicine, University of North Texas Health Science Center, Fort Worth, TX 76107, USA

${ }^{7}$ Institute for Aging and Alzheimer's Disease Research, University of North Texas Health Sciences Center, Fort Worth, TX 76107, USA

Correspondence should be addressed to Valerie H. Balldin, valerielhobson@yahoo.com

Received 19 April 2012; Revised 25 July 2012; Accepted 3 August 2012

Academic Editor: Christoph Laske

Copyright ( $) 2012$ Valerie H. Balldin et al. This is an open access article distributed under the Creative Commons Attribution License, which permits unrestricted use, distribution, and reproduction in any medium, provided the original work is properly cited.

\begin{abstract}
Background. Considerable research documents an association between pro- and anti-inflammatory markers and Alzheimer's disease $(\mathrm{AD})$, yet the differential relation between these markers and neuropsychological functioning in $\mathrm{AD}$ and nondemented controls has received less attention. The current study sought to evaluate the relationship between peripheral markers of inflammation (both pro- and anti-inflammatory) and neuropsychological functioning through the Texas Alzheimer's Research and Care Consortium (TARCC) cohort. Methods. There were 320 participants (Probable AD $n=124$, Controls $n=196$ ) in the TARCC Longitudinal Research Cohort available for analysis. Regression analyses were utilized to examine the relation between proinflammatory and anti-inflammatory markers and neuropsychological functioning. Follow-up analyses were conducted separately by case versus control status. Results. Proinflammatory and anti-inflammatory markers were found to be associated with neuropsychological testing. Third tertile proinflammatory markers were negatively associated with measures of attention and language, and anti-inflammatory markers were positively associated with measures of immediate verbal memory and delayed verbal and visual memory. Conclusions. These findings support the link between peripheral inflammatory markers and neuropsychological functioning and suggest the utility of examining profiles of inflammatory markers in the future.
\end{abstract}

\section{Introduction}

Inflammation has been hypothesized to modulate a number of pathogenic processes that are associated with Alzheimer's disease $(\mathrm{AD})$, including the amyloid cascade [1-3], although the underlying etiology and full nature of the relationship remains unclear. Both proinflammatory and anti-inflammatory proteins have been shown to be stimulated by injury, $\beta$-amyloid toxicity, and ischemia [4], and inflammation is increased in both hypertension and atherosclerosis [5]. Furthermore, peripheral inflammation has been related to poorer cognitive performance [6-9], as well as cognitive decline [10], although these findings have not always been consistently supported [11].
Several large population-based studies have examined serum markers of inflammation and their relation to cognitive functioning. The Honolulu Asia Aging Study (HAAS) [12] found that raised levels of C-reactive protein (CRP; an inflammatory marker) in mid-life was associated with a significantly increased risk for vascular dementia (VaD) and $\mathrm{AD}$, with or without the presence of cardiovascular disease (CVD). The Rotterdam Study reported a link between CRP risk for $\mathrm{AD}$ and $\mathrm{VaD}$. Additionally, it has been proposed that individuals with cognitive impairment may have a different balance of proinflammatory and anti-inflammatory cytokines compared to those with normal aging $[13,14]$.

While considerable research has documented that chronic inflammation and high levels of proinflammatory 
cytokines are fundamental components of $\mathrm{AD}$, the relationship between these markers and decline in specific neuropsychological performance has received less attention. Research in this area has tended to use brief measures of global cognitive functioning (e.g., mini-mental state examination (MMSE), Short Portable Mental Questionnaire (SPMQ) making it difficult to gain insight into focal cognitive deficits that may be associated with elevated cytokine levels [15].

The current project sought to examine the link between a number of pro- and anti-inflammatory markers and neuropsychological functioning among a sample of patients diagnosed with $\mathrm{AD}$ and cognitively normal controls from the Texas Alzheimer's Research and Care Consortium (TARCC) research. It was hypothesized that there would be a significant relationship between serum levels of inflammatory cytokines (a net increase in the proinflammatory profile) and performance on tests of memory and verbal fluency compared to other neuropsychological domains.

\section{Materials and Methods}

Participants. Participants included 320 individuals (124 diagnosed with Probable AD and 196 normal controls) enrolled the TARCC Longitudinal Research Cohort. The methodology of the TARCC project has been described in detail elsewhere [16]. Briefly, each participant undergoes an annual evaluation that includes a medical examination, interview, neuropsychological testing, and blood draw. All patients met consensus-based diagnosis for probable $\mathrm{AD}$ based on NINCDS-ADRDA criteria [17] and controls performed within normal limits on psychometric assessment and were assigned a Clinical Dementia Rating scale (CDR) global score of 0.0. AD patients were included if their CDR global scores were .5 or $1.0 ; 46$ had CDR global scores of 0.5 and 78 had scores of 1.0. The TARCC project received institutional review board approval and all participants and/or caregivers provided written informed consent.

\section{Measures}

The TARCC neuropsychology core battery consists of neuropsychological instruments administered as part of the established Alzheimer's disease clinical/research platforms at each participating institution and included digit span (WAIS-R, WAIS-III, WMS-R), Trail Making Test, WMS Logical Memory (WMS-R and WMS-III), Boston Naming Test (30- and 60-item versions), verbal fluency (FAS), Clock Drawing Test, the American National Adult Reading Test (AMNART), the Geriatric Depression Scale (GDS-30), minimental state examination (MMSE) [18], and the Clinical Dementia Rating scale (CDR) [19]. In order to equate scores from digit span and story memory scales, all raw scores were converted to scale scores based on previously published normative data [20]. For the Boston Naming Test, the current group recently conducted an independent study that demonstrated the psychometric properties of an estimated 60-item BNT score that can be calculated from 30-item versions [21]. Adjusted scale scores were utilized as dependent variables in analyses.
TABLE 1: Coefficient of variation by marker.

\begin{tabular}{lccc}
\hline Marker & Combined & AD & Control \\
\hline TNFalpha & 58.18 & 54.55 & 59.68 \\
A1A & 49.24 & 44.44 & 53.13 \\
IL8 & 11.38 & 10.29 & 12.21 \\
CRP & 190.02 & 317.43 & 139.39 \\
IL-1ra & 15.04 & 15.54 & 14.15 \\
IL10 & 31.55 & 27.96 & 32.98 \\
\hline
\end{tabular}

A1A: alpha-1 antitrypsin; CRP: C-reactive protein.

\section{Assays}

Nonfasting samples were collected in serum-separating tubes during clinical evaluations, allowed to clot at room temperature for thirty minutes, centrifuged, aliquoted, and stored at $-80^{\circ} \mathrm{C}$ in plastic vials. Serum samples were sent frozen to Rules-Based Medicine (http://www.rulesbasedmedicine.com/, Austin, TX, USA) where they were thawed for assay without additional freeze-thaw cycles. RulesBased Medicine conducted multiplexed immunoassay via their human multianalyte profile (human MAP). Multiple proteins, including proinflammatory markers (TNFalpha, Alpha1Antitrypsin, IL-8, and C-reactive protein) and antiinflammatory markers (IL-1ra, IL-10), were quantified though multiplex fluorescent immunoassay utilizing colored microspheres with protein-specific antibodies. The coefficient of variation (CV), a normalized measure of dispersion of a probability distribution ( $\mathrm{CV}=$ standard deviation/mean), for each marker (run only once) are shown in Table 1 . The proinflammatory and anti-inflammatory markers were chosen due to past research showing their reliability in use in the study of inflammatory processes [22-26].

Additional information regarding assay performance can be found online. Assays conducted by this company utilizing this platform, including TARCC data, have been published elsewhere [27-29]. For additional information, please refer to (http://www.rulesbasedmedicine.com/).

\section{Analyses}

Statistical analyses were conducted using SPSS version 18.0 (SPSS, Chicago, IL). Comparisons between NC and AD were conducted using $t$-tests for continuous measures and $\chi^{2}$ for categorical measures. The relation between inflammatory makers and cognitive test performance was examined via a series of regression models for all participants $(\mathrm{AD}$ and NC) with proinflammatory and anti-inflammatory proteins entered in separate blocks as predictor variables and neuropsychological test scores entered as outcome variables. Levels of inflammatory proteins were categorized into tertiles based on the distribution of the variable (i.e., 1st, 2nd, and 3rd group). The number of markers (pro- and antiinflammatory separately) in the third tertile for each marker was summed for each participant and this summed score was entered as a predictor variable into a linear regression model with neuropsychological test scores as outcome variables (i.e., if the participant's value for IL- 8 and IL-10 were in 
TABle 2: Demographics by diagnosis.

\begin{tabular}{lccr}
\hline & AD & Control & $P$ value \\
\hline $\begin{array}{l}\text { Number of subjects } \\
\text { Sex }\end{array} \quad 124$ & 196 & - \\
$\quad$ Female & $77(62.1 \%)$ & $134(68.4 \%)$ & 0.25 \\
Age (years) & & & $<0.01$ \\
$\quad$ Mean (SD) & $76.72(7.80)$ & $70.47(8.81)$ & \\
$\quad$ Range & $57-91$ & $55-90$ & $<0.01$ \\
Education (years) & $14.35(3.30)$ & $15.52(2.73)$ & $10-25$ \\
$\quad$ Mean (SD) & $5-22$ & $50(25.8 \%)$ & $<0.01$ \\
$\quad$ Range & & & \\
ApoE & $71(61.7 \%)$ & & \\
$\quad$ One or more E4 & &
\end{tabular}

the third tertile, the participant had a " 2 " entered as the predictor variable into the regression model).

Follow-up analyses were then conducted examining the relationship between proinflammatory and anti-inflammatory markers and neuropsychological functioning on case status ( $\mathrm{NC}$ versus $\mathrm{AD}$ ). All biomarker results were transformed using $\log$ base 10 in order to evenly distribute the scores of the markers. Assumptions for all statistics tests were checked for violations and statistical significance was declared for $P$ value $<0.01$.

\section{Results}

Average age and education of the control group was $70.5 \pm 8.8$ and $15.5 \pm 2.7$, respectively, while average age and education of the $\mathrm{AD}$ group was $76.7 \pm 7.8$ and $14.4 \pm 3.3$, respectively. The sample was over $95 \%$ Caucasian, and there were more females than males, though gender distribution was not significantly different between groups. As expected, $\mathrm{AD}$ patients obtained significantly lower MMSE scores (mean = $22.3 \pm 4.1$, median $=23.0)$ than controls $($ mean $=29.4 \pm 0.9$, median $=30.0)$ as well as higher CDR sum of boxes scores $(\mathrm{AD}$ mean $=5.2 \pm 2.3$, median $=5$; controls mean $=0.0 \pm 0.04$, median $=0.0)$. Demographic characteristics of the study population are shown in Table 2.

\section{Individual Markers and Neuropsychological Functioning}

7.1. Proinflammatory Markers. Higher IL-8 levels were associated with significantly poorer scores in global cognition (MMSE $\beta=-0.185, P=0.002$ ), as well as immediate visual memory (WMS-III Visual Reproduction I $\beta=-0.157$, $P=0.009$ ), and verbal fluency (COWAT $\beta=-0.172, P=$ $0.004)$. Higher IL-8 levels were also associated with significantly greater disease severity (CDR Sum of Boxes; $\beta=0.205$, $P<0.001)$.

Higher CRP levels were associated with significantly greater disease severity (CDR Sum of Boxes; $\beta=-0.201$, $P=0.001)$. However, elevated CRP levels were also related to significantly better global cognition (MMSE; $\beta=0.533, P=$
0.006), verbal (WMS-III Logical Memory I; $\beta=0.202, P=$ 0.001; WMS-III Logical Memory II; $\beta=0.712, P=0.001$ ), and delayed visual memory (WMS-III Visual Reproduction II; $\beta=0.710, P<0.001$ ).

Higher TNF-alpha levels were associated with better delayed verbal (WMS-III Logical Memory II; $\beta=0.178$, $P=0.007$ ) and immediate visual memory (WMS-III Visual Reproduction I; $\beta=0.196, P=0.002$ ). Neuropsychological tests by diagnosis are shown in Table 3 .

7.2. Anti-Inflammatory Markers. Higher IL-1ra levels were associated with disease severity (CDR Sum of boxes; $\beta=$ $-0.161, P=0.004$ ), better scores in verbal memory (WMSIII Logical Memory I; $\beta=0.168, P=0.005$; WMS-III Logical Memory II; $\beta=0.164, P=0.006$ ), and visual memory (WMS-III Visual Reproduction I; $\beta=0.180, P=0.002$; WMS-III Visual Reproduction II; $\beta=0.180, P=0.002$ ).

\section{Summed Number of Markers by Tertiles and Separated by Diagnostic Category}

The sum of the number of proinflammatory markers in the $3^{\text {rd }}$ tertile was found to be associated with significantly better information processing speed (Trails $\mathrm{A} ; \beta=0.177$, $P=0.004)$. When looking at AD cases only, the sum of the proinflammatory markers was associated with significantly better immediate verbal memory (WMS-III Logical Memory I; $\beta=0.163, P=0.004$ ), but poorer fluency (COWAT; $\beta=-0.173, P=0.010)$. In the NC group, the sum of the proinflammatory markers was associated with significantly better processing speed (Trails A; $\beta=0.165, P=0.010$ ). See Table 4 for biomarkers by diagnosis.

\section{Discussion}

The current study demonstrates that the association between inflammatory markers is quite complex. In our sample, proinflammatory markers were found to be both positively associated with immediate and delayed verbal and visual memory, and disease severity and global cognition and negatively associated with measures of immediate visual memory, 
TABle 3: Neuropsychological testing by diagnosis.

\begin{tabular}{|c|c|c|c|}
\hline & $\mathrm{AD}$ & Control & $P$ value \\
\hline Number of subjects & 124 & 196 & - \\
\hline \multicolumn{4}{|l|}{ MMSE } \\
\hline Mean (SD) & $22.32(4.13)$ & $29.43(0.88)$ & \multirow{2}{*}{$<0.01$} \\
\hline Range & $8-30$ & $26-30$ & \\
\hline \multicolumn{4}{|l|}{ CDR sum of boxes } \\
\hline Mean (SD) & $5.15(2.30)$ & $0.00(0.04)$ & \multirow{2}{*}{$<0.01$} \\
\hline Range & $0.5-13.0$ & $0.0-0.5$ & \\
\hline \multicolumn{4}{|l|}{ Trails A } \\
\hline Mean (SD) & $6.89(2.86)$ & $10.31(2.68)$ & \multirow{2}{*}{$<0.01$} \\
\hline Range & $2-14$ & $2-17$ & \\
\hline \multicolumn{4}{|l|}{ Trails B } \\
\hline Mean (SD) & $5.27(3.42)$ & $10.96(2.53)$ & \multirow{2}{*}{$<0.01$} \\
\hline Range & $2-18$ & $3-18$ & \\
\hline \multicolumn{4}{|c|}{ WMS-III Logical Memory I } \\
\hline Mean (SD) & $4.64(2.46)$ & $13.57(2.76)$ & \multirow{2}{*}{$<0.01$} \\
\hline Range & $1-10$ & $6-18$ & \\
\hline \multicolumn{4}{|c|}{ WMS-III Logical Memory II } \\
\hline Mean (SD) & $3.69(1.89)$ & $13.99(2.63)$ & \multirow{2}{*}{$<0.01$} \\
\hline Range & $1-11$ & $5-19$ & \\
\hline \multicolumn{4}{|c|}{ WMS-III Visual Reproduction I } \\
\hline Mean (SD) & $4.97(2.77)$ & $12.41(3.19)$ & \multirow{2}{*}{$<0.01$} \\
\hline Range & $1-13$ & $5-18$ & \\
\hline \multicolumn{4}{|c|}{ WMS-III Visual Reproduction II } \\
\hline Mean (SD) & $4.82(2.27)$ & $13.57(3.14)$ & \multirow{2}{*}{$<0.01$} \\
\hline Range & $2-12$ & $5-19$ & \\
\hline \multicolumn{4}{|l|}{ Boston naming test } \\
\hline Mean (SD) & $7.32(3.79)$ & $11.94(3.04)$ & \multirow{2}{*}{$<0.01$} \\
\hline Range & $2-16$ & $2-17$ & \\
\hline \multicolumn{4}{|l|}{ COWAT } \\
\hline Mean (SD) & $7.91(2.89)$ & $11.64(2.75)$ & \multirow{2}{*}{$<0.01$} \\
\hline Range & $2-18$ & $4-18$ & \\
\hline \multicolumn{4}{|l|}{ AMNART } \\
\hline Mean (SD) & $9.28(3.55)$ & $12.11(3.37)$ & \multirow{2}{*}{$<0.01$} \\
\hline Range & $2-18$ & $2-18$ & \\
\hline \multicolumn{4}{|l|}{ Estimated VIQ } \\
\hline Mean (SD) & $108.32(15.05)$ & $117.12(9.88)$ & \multirow{2}{*}{$<0.01$} \\
\hline Range & $38.66-127.44$ & $42.11-132.28$ & \\
\hline
\end{tabular}

verbal fluency, and global cognition. The anti-inflammatory markers were found to be significantly positively and negatively associated with a measure of global cognition and disease severity (CDR), and immediate and delayed visual and verbal memory. These findings are not surprising as such conflicting results have been documented previously in the literature. These findings also make sense biologically as these immune-related markers serve a purpose that only becomes detrimental when the system gets out of balance, which has been observed in $\mathrm{AD}$. Our findings also point to the fact that the relationship between inflammation and neuropsychological functioning varies according to disease status and cognitive domain. In fact, the majority of our significant findings were for $\mathrm{AD}$ cases rather than controls.
When examined by case status, proinflammatory markers were negatively associated with a measure of verbal fluency and positively associated with immediate verbal memory for the AD group. Within the normal control group, proinflammatory markers were positively associated with processing speed. In addition, a positive association with information processing speed was observed among individuals in the 3rd tertile of proinflammatory markers.

These findings suggest the importance of examining profiles of the inflammatory response. As suggested in prior research, [30] those with cognitive impairment may possess different proinflammatory and anti-inflammatory profiles compared to those without cognitive impairment. For example, the Leiden $85+$ study examined the ratio of TNFalpha, 
TABLE 4: Biomarkers by diagnosis.

\begin{tabular}{|c|c|c|c|}
\hline & $\mathrm{AD}$ & Control & $P$ value \\
\hline Number of subjects & 124 & 196 & - \\
\hline \multicolumn{4}{|l|}{ TNF-alpha Log } \\
\hline Mean (SD) & $0.55(0.30)$ & $0.62(0.37)$ & \multirow{2}{*}{0.05} \\
\hline Range & $-0.30-1.04$ & $-0.30-1.79$ & \\
\hline \multicolumn{4}{|c|}{ Alpha-1 Antitrypsin Log } \\
\hline Mean (SD) & $0.36(0.16)$ & $0.32(0.17)$ & \multirow{2}{*}{0.09} \\
\hline Range & $-0.23-0.71$ & $-0.43-0.71$ & \\
\hline \multicolumn{4}{|l|}{ IL-8 Log } \\
\hline Mean (SD) & $1.36(0.14)$ & $1.31(0.16)$ & \multirow{2}{*}{$<0.01$} \\
\hline Range & $1.04-1.78$ & $0.98-1.84$ & \\
\hline \multicolumn{4}{|c|}{ C-Reactive Protein Log } \\
\hline Mean (SD) & $0.14(0.52)$ & $0.33(0.46)$ & \multirow{2}{*}{$<0.01$} \\
\hline Range & $-1.34-1.36$ & $-1.19-1.40$ & \\
\hline \multicolumn{4}{|l|}{ IL-1ra Log } \\
\hline Mean (SD) & $1.93(0.30)$ & $2.05(0.29)$ & \multirow{2}{*}{$<0.01$} \\
\hline Range & $0.90-2.61$ & $0.90-2.62$ & \\
\hline \multicolumn{4}{|l|}{ IL-10 Log } \\
\hline Mean (SD) & $0.93(0.26)$ & $0.94(0.31)$ & \multirow{2}{*}{0.83} \\
\hline Range & $0.04-1.43$ & $0.04-1.62$ & \\
\hline \multicolumn{4}{|l|}{ Sum of markers } \\
\hline Mean (SD) & $2.40(1.85)$ & $2.76(1.95)$ & \multirow{2}{*}{0.11} \\
\hline Range & $0-8$ & $0-8$ & \\
\hline
\end{tabular}

a proinflammatory cytokine and IL-10, an anti-inflammatory cytokine. The ratio was higher in patients diagnosed with $\mathrm{AD}$ compared with normal controls which suggests a net "proinflammatory profile". The findings of the current study also indicate the importance of examining the combination of upper median levels of pro- and anti-inflammatory biomarkers.

The current findings are limited by the cross-sectional nature of the analyses; however, the TARC cohort is being evaluated annually and follow-up analyses examining the profiles of anti- and proinflammatory markers in those with and without a diagnosis of $\mathrm{AD}$ will be completed. The current findings point towards the use of profiles of biomarkers as a possible way to understand the relationship between inflammation and neuropsychological functioning versus the standard approach of examining biomarkers individually.

\section{Authors' Contributions}

VB conceived the study, drafted the paper and took lead on design and coordination of the paper. $\mathrm{RB}, \mathrm{SO}$, and $\mathrm{JH}$ provided considerable aid in conceiving this study, drafting the paper and providing edits. LH carried out all statistical analyses and provided edits of paper. RDA participated in the design of the study and provided edits to the paper.

\section{Acknowledgments}

Research reported in this publication was supported by the National Institute on Aging of the National Institutes of
Health under Award no. R01AG039389 and P30AG12300. The content is solely the responsibility of the authors and does not necessarily represent the official views of the National Institutes of Health. This study was made possible by the Texas Alzheimer's Research and Care Consortium (TARCC) funded by the state of Texas through the Texas Council on Alzheimer's Disease and Related Disorders. The authors would like to thank all of the participants of the TARCC along with the incredible support staff that make this study possible. Investigators from the Texas Alzheimer's Research and Care Consortium: Baylor College of Medicine: Rachelle Doody MD, PhD, Susan Rountree MD, Valory Pavlik PhD, Wen Chan PhD, Paul Massman PhD, Eveleen Darby, Tracy Evans RN, Aisha Khaleeq; Texas Tech University Health Science Center; Gregory Schrimsher, PhD, Andrew Dentino, MD, Ronnie Orozco; University of North Texas Health Science Center: Thomas Fairchild, PhD, Janice Knebl, DO, Douglas Mains, Lisa Alvarez, Erin Braddock, Rosemary McCallum, Leigh Johnson; University of Texas Southwestern Medical Center: Perrie Adams, PhD, Roger Rosenberg, MD, Myron Weiner, MD, Benjamin Williams, MD, PhD, Mary Quiceno, MD, Joan Reisch, PhD, Ryan Huebinger, PhD, Guanghua Xiao, PhD, Doris Svetlik, Amy Werry, Janet Smith; University of Texas Health Science Center-San Antonio: Donald Royall, MD, Raymond Palmer, PhD, Marsha Polk.

\section{References}

[1] R. W. Keefover, E. D. Rankin, P. M. Keyl, J. C. Wells, J. Martin, and J. Shaw, "Dementing illnesses in rural populations: the need for research and challenges confronting investigators," Journal of Rural Health, vol. 12, no. 3, pp. 178-187, 1996. 
[2] J. Sundelöf, L. Kilander, J. Helmersson et al., "Systemic inflammation and the risk of Alzheimer's disease and dementia: a prospective population-based study," Journal of Alzheimer's Disease, vol. 18, no. 1, pp. 79-87, 2009.

[3] H. Johnston, H. Boutin, and S. M. Allan, "Assessing the contribution of inflammation in models of Alzheimer's disease," Biochemical Society Transactions, vol. 39, no. 4, pp. 886-890, 2011.

[4] E. N. Benveniste, "Inflammatory cytokines within the central nervous system: sources, function, and mechanism of action," American Journal of Physiology, vol. 263, no. 1, pp. C1-C16, 1992.

[5] J. Kaye, Y. Michael, J. Calvert, M. Leahy, D. Crawford, and P. Kramer, "Exceptional brain aging in a rural population-based cohort," Journal of Rural Health, vol. 25, no. 3, pp. 320-325, 2009.

[6] R. P. Friedland, "Epidemiology, education, and the ecology of Alzheimer's disease," Neurology, vol. 43, no. 2, pp. 246-249, 1993.

[7] M. Ganguli, H. H. Dodge, P. Chen, S. Belle, and S. T. DeKosky, "Ten-year incidence of dementia in a rural elderly US community population. The MoVIES project," Neurology, vol. 54, no. 5, pp. 1109-1116, 2000.

[8] J. R. Harvan and V. Cotter, "An evaluation of dementia screening in the primary care setting," Journal of the American Academy of Nurse Practitioners, vol. 18, no. 8, pp. 351-360, 2006.

[9] A. L. Jefferson, J. M. Massaro, A. S. Beiser et al., "Inflammatory markers and neuropsychological functioning: the framingham heart study," Neuroepidemiology, vol. 37, no. 1, pp. 21-30, 2011.

[10] P. Komulainen, T. A. Lakka, M. Kivipelto et al., "Serum high sensitivity C-reactive protein and cognitive function in elderly women," Age and Ageing, vol. 36, no. 4, pp. 443-448, 2007.

[11] J. Weuve, P. M. Ridker, N. R. Cook, J. E. Buring, and F. Grodstein, "High-sensitivity C-reactive protein and cognitive function in older women," Epidemiology, vol. 17, no. 2, pp. 183-189, 2006.

[12] R. Schmidt, H. Schmidt, J. D. Curb, K. Masaki, L. R. White, and L. J. Launer, "Early inflammation and dementia: a 25year follow-up of the Honolulu-Asia Aging Study," Annals of Neurology, vol. 52, no. 2, pp. 168-174, 2002.

[13] A. M. Bodles and S. W. Barger, "Cytokines and the aging brainwhat we don't know might help us," Trends in Neurosciences, vol. 27, no. 10, pp. 621-626, 2004.

[14] C. A. Murray and M. A. Lynch, "Evidence that increased hippocampal expression of the cytokine interleukin- $1 \beta$ is a common trigger for age- and stress-induced impairments in longterm potentiation," Journal of Neuroscience, vol. 18, no. 8, pp. 2974-2981, 1998.

[15] R. S. Wilson, D. A. Evans, J. L. Bienias, C. F. Mendes De Leon, J. A. Schneider, and D. A. Bennett, "Proneness to psychological distress is associated with risk of Alzheimer's disease," Neurology, vol. 61, no. 11, pp. 1479-1485, 2003.

[16] S. Waring, S. E. O'Bryant, J. S. Reisch et al., “The Texas Alzheimer's Research Consortium longitudinal research cohort: study design and baseline characteristics," Texas Public Health Journal, vol. 60, no. 3, pp. 9-13, 2008.

[17] G. McKhann, D. Drachman, and M. Folstein, "Clinical diagnosis of Alzheimer's disease: report of the NINCDS-ADRDA work group under the auspices of Department of Health and Human Services Task Force on Alzheimer's disease," Neurology, vol. 34, no. 7, pp. 939-944, 1984.

[18] M. F. Folstein, S. E. Folstein, and P. R. McHugh, “'Mini mental state'. A practical method for grading the cognitive state of patients for the clinician," Journal of Psychiatric Research, vol. 12, no. 3, pp. 189-198, 1975.

[19] C. P. Hughes, L. Berg, and W. L. Danziger, "A new clinical scale for the staging of dementia," British Journal of Psychiatry, vol. 140, no. 6, pp. 566-572, 1982.

[20] B. A. Steinberg, L. A. Bieliauskas, G. E. Smith, and R. J. Ivnik, "Mayo's older Americans normative studies: age- and IQadjusted norms for the Trail-Making Test, the Stroop Test, and MAE Controlled Oral Word Association Test," Clinical Neuropsychologist, vol. 19, no. 3-4, pp. 329-377, 2005.

[21] V. L. Hobson, J. R. Hall, M. Harvey et al., "An examination of the Boston Naming Test: calculation of "estimated" 60-item score from 30- and 15-item scores in a cognitively impaired population," International Journal of Geriatric Psychiatry, vol. 26, no. 4, pp. 351-355, 2011.

[22] Y. Yu, "Hepatitis B virus induces a novel inflammation network involving three inflammatory factors, IL-29, IL-8, and Cyclooxygenase-2," The Journal of Immunology (1950), vol. 187, no. 9, pp. 4844-4860, 2011.

[23] B. E. Barton, "IL-6-like cytokines and cancer cachexia: consequences of chronic inflammation," Immunologic Research, vol. 23, no. 1, pp. 41-58, 2001.

[24] K. Marinou, "Biomarkers predicting metabolic syndrome: the role of adiponectin and systemic inflammation," International Journal of Cardiology, vol. 155, no. 2, pp. 286-287, 2012.

[25] J. Dong, E. Jimi, C. Zeiss, M. S. Hayden, and S. Ghosh, "Constitutively active NF- $\kappa \mathrm{B}$ triggers systemic TNF $\alpha$-dependent inflammation and localized TNF $\alpha$-independent inflammatory disease," Genes and Development, vol. 24, no. 16, pp. 17091717, 2010.

[26] J. M. Dayer, R. Chicheportiche, C. Juge-Aubry, and C. Meier, "Adipose tissue has anti-inflammatory properties: focus on IL-1 receptor antagonist (IL-1Ra)," Annals of the New York Academy of Sciences, vol. 1069, pp. 444-453, 2006.

[27] M. P. Doescher, J. E. Jackson, A. Jerant, and L. Gary Hart, "Prevalence and trends in smoking: a national rural study," Journal of Rural Health, vol. 22, no. 2, pp. 112-118, 2006.

[28] R. N. Jones and J. J. Gallo, "Dimensions of the Mini-Mental State Examination among community dwelling older adults," Psychological Medicine, vol. 30, no. 3, pp. 605-618, 2000.

[29] S. E. O'Bryant, J. D. Humphreys, G. E. Smith et al., "Detecting dementia with the mini-mental state examination in highly educated individuals," Archives of Neurology, vol. 65, no. 7, pp. 963-967, 2008.

[30] E. Van Exel, A. J. M. De Craen, E. J. Remarque et al., "Interaction of atherosclerosis and inflammation in elderly subjects with poor cognitive function," Neurology, vol. 61, no. 12, pp. 1695-1701, 2003. 


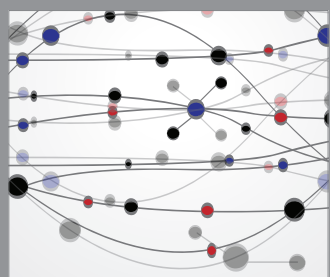

The Scientific World Journal
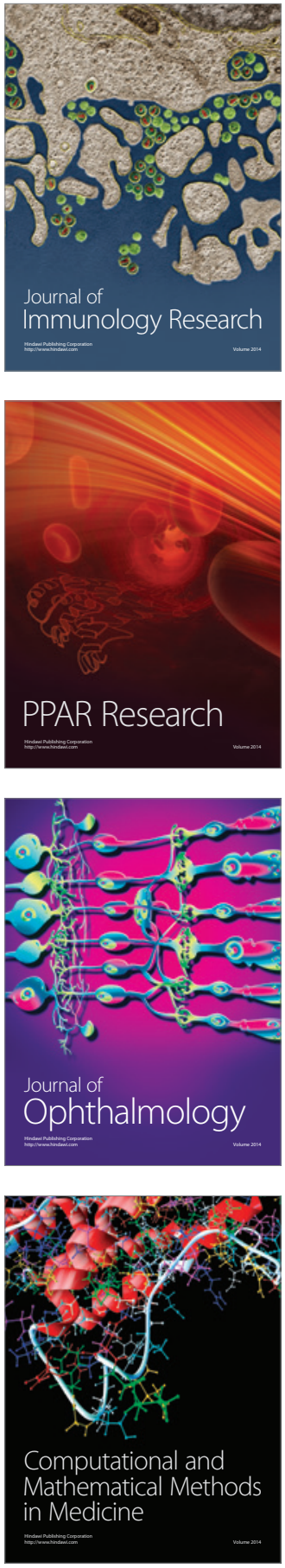

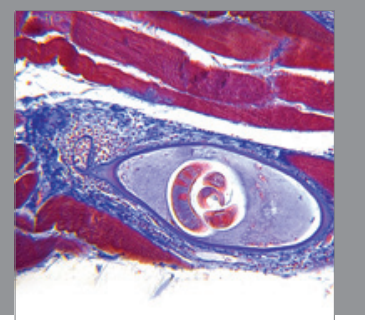

Gastroenterology

Research and Practice
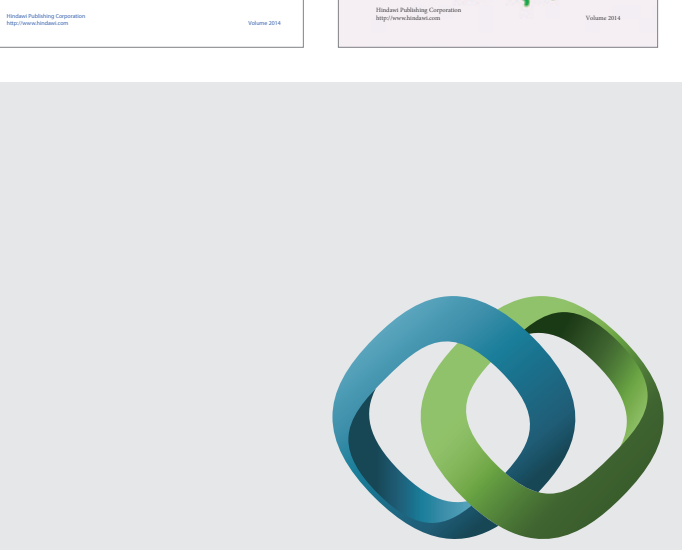

\section{Hindawi}

Submit your manuscripts at

http://www.hindawi.com
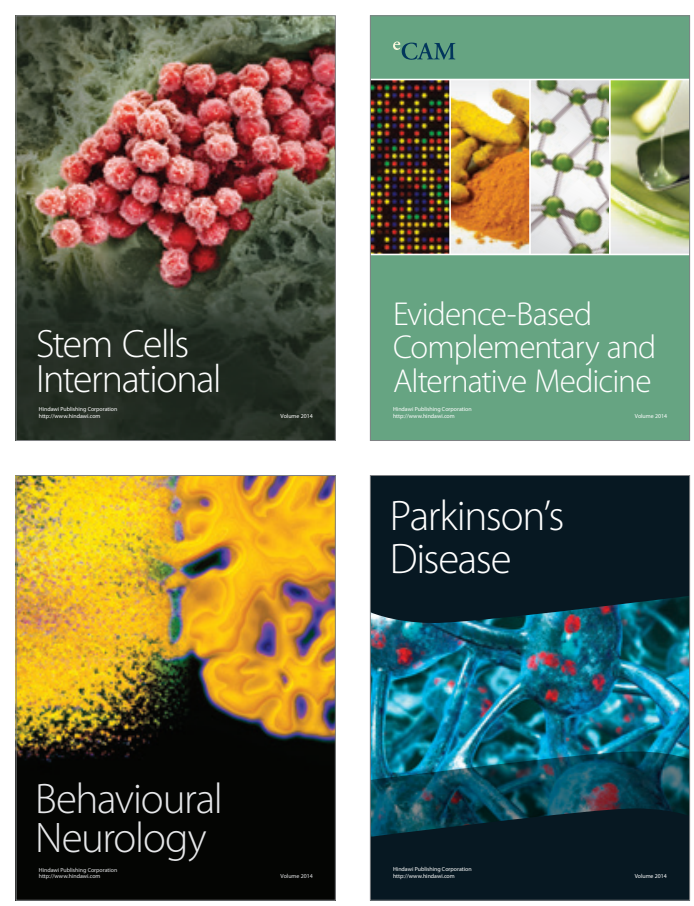

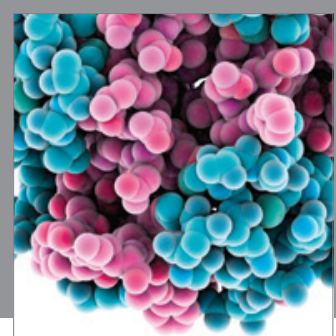

Journal of
Diabetes Research

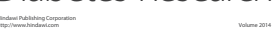

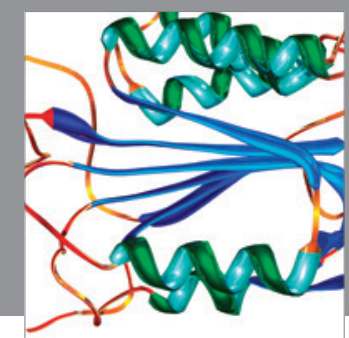

Disease Markers
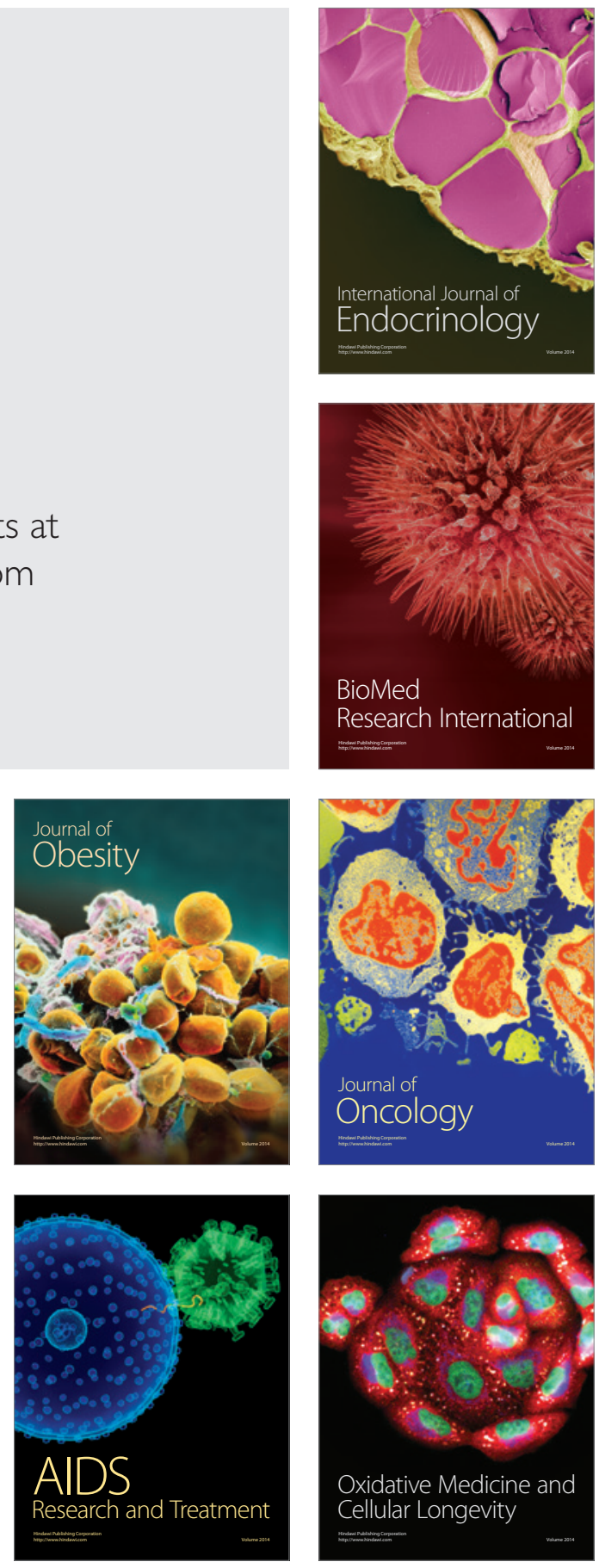